\section{Ejercicio intermitente y consejería nutricional mejoran control glicémico y calidad de vida en pacientes con diabetes mellitus tipo 2}

\author{
PEDRO MANGIAMARCHI ${ }^{1, \mathrm{a}}$, ALEXIS CANIUQUEO ${ }^{2, \mathrm{~b}}$, \\ RODRIGO RAMÍREZ-CAMPILLO ${ }^{3, \mathrm{c}}$, PATRICIO CÁRDENAS ${ }^{4}$, \\ SYLVANA MORALES ${ }^{5, \mathrm{~d}}$, JOHNATTAN CANO-MONTOYA ${ }^{6, \mathrm{e}}$, \\ GUILHERME BRESCIANI ${ }^{7, f}$, CRISTIAN ÁLVAREZ ${ }^{3, g}$
}

Background: High-intensity interval training (HIIT) improves cardiometabolic markers, but its effects on the quality of life of patients with type 2 diabetes (T2D) is not well known. Aim: To determine the effects of a 12-week HIIT exercise program on cardiometabolic and quality of life variables of T2D patients. Material and Methods: Nine T2D women were assigned to a HIIT + nutritional education (GE) and 10, to a nutritional education alone group (GC). At baseline and after each intervention, anthropometric and body composition parameters using bio-impedance were assessed, and a blood sample was obtained to measure serum lipid levels, blood glucose and glycated hemoglobin. Quality of life was assessed using the SF-12 questionnaire adapted for the Chilean population. Results: There were no significant changes on the lipid profile variables in the GE group, although HDL cholesterol was increased significantly $(p<0.05)$ in the $G C$ group. Total fat mass was decreased in the GE group from $43.5 \pm 1.5$ to $41.9 \pm 1.5 \%, p<0.01$. Fasting glucose and glycated hemoglobin decreased in the GE group. There was a significant correlation between the decrease in total fat mass and that of glycated hemoglobin. There were significant increases in quality of life parameters; physical function, physical role, pain, general health, vitality, emotional role, mental health, and social function in the GE but not in the GC group. Conclusions: A 12-week program of HIIT plus nutritional education improves cardiometabolic and quality of life parameters on type 2 diabetics.

(Rev Med Chile 2017; 145: 845-853)

Key words: Blood Glucose; Exercise; Diabetes Mellitus, Type 2; Quality of Life.

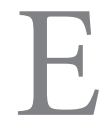

n el año 2010, entre 221 y 347 millones de personas fueron diagnosticadas con diabetes mellitus a nivel mundial ${ }^{1}$, y al año 2014, 8,5\% de personas mayores de 18 años presentó diabetes mellitus ${ }^{2}$. Los tipos de diabetes mellitus
'Centro Comunitario de Rehabilitación, Padre las Casas, Chile.

2Laboratorio de Fisiología y

Biomecánica, Universidad Autónoma

de Chile, Temuco, Chile.

${ }^{3}$ Departamento de Ciencias de

la Actividad Física, Núcleo de

Investigación en Salud, Actividad Física

y Deporte, Universidad de los Lagos,

Osorno, Chile.

${ }^{4}$ Unidad de Medicina Interna. Hospital

Dr. Hernán Henríquez Aravena.

Temuco, Chile.

${ }^{5}$ Centro de Salud Familiar las Colinas,

Padre las Casas, Chile.

${ }^{6}$ Escuela de Kinesiología, Instituto de Aparato Locomotor y Rehabilitación,

Facultad de Medicina, Universidad

Austral de Chile. Valdivia, Chile.

${ }^{7}$ Grupo IRyS. Escuela de Educación

Física. Pontificia Universidad Católica

de Valparaíso, Chile.

aProfesor de Educación física, Msc. en Actividad Física y Salud.

bProfesor de Educación Física. PhD en Ciencias de la Motricidad Humana.

'Profesor de Educación Física, PhD en Ciencias de la Salud.

dEnfermera E.U.

eKinesiólogo, Msc Fisiología Clínica del Ejercicio.

fLicenciado en Educación Física, PhD en Ciencias de la Actividad Física.

gProfesor de Educación Física, PhDC en Ciencias de la Salud.

Este proyecto de investigación, fue financiado con los fondos del programa de salud cardiovascular del departamento de salud, y con los recursos del Centro Comunitario de Rehabilitación de Padre las Casas, IX Región de la Araucanía, Temuco, Chile. No se declaran conflictos de intereses entre las fuentes de financiamiento

Recibido el 12 de abril de 2017, aceptado el 21 de agosto de 2017.

Correspondencia a:

Cristian Alvarez, Msc., PhD ( )

Departamento de Ciencias de la Actividad Física, Universidad de Los Lagos, Campus Chuyaca, Osorno, Chile. más conocidas son la diabetes mellitus tipo 1, tipo 2 (DM2) y diabetes gestacional. La DM2 es más prevalente, incluyendo 90 a $95 \%$ de todos los casos de diabetes ${ }^{3}$. En Chile, la Encuesta Nacional de Salud reportó que 9,4\% de la población padece 
DM2, con una mayor prevalencia en personas con menor nivel socio económico/educacional ${ }^{4}$, quienes tienen un estilo de vida no saludable y donde la falta de actividad física (AF) es el principal factor regulador de tal condición ${ }^{5}$.

Por otra parte, debido a que, a) la respuesta cardiovascular como la frecuencia cardiaca (FC) tiende a exacerbarse ante ejercicios de tipo "continuos", produciendo ello un mayor estrés cardiovascular ${ }^{6}$ y b) considerando la excusa de "falta de tiempo" de la población para no adherir a las recomendaciones internacionales de AF $(150 / 300 \text { min de AF por semana })^{7,8}$, se ha incrementado la práctica de ejercicios de tipo tiempo-eficiente como el ejercicio intermitente de alta intensidad o HIIT, por las siglas del nombre en inglés, High-intensity interval training. Dicha característica de tiempo-eficiencia ha sido demostrada en estudios donde se ha contrastado el HIIT con modelos de ejercicio continuo ${ }^{9}$. El HIIT ha demostrado efectos benéficos en parámetros metabólicos como la hemoglobina glicosilada $(\mathrm{HbAlc})^{10}$, glicemia ${ }^{11,12}$, composición corporal ${ }^{13}$, presión sanguínea ${ }^{14}$ y la condición física ${ }^{12}$. Estos cambios se inician a nivel molecular, como el incremento en la densidad mitocondrial y los trasportadores de glucosa GLUT- $4^{11}$.

Sin embargo, el reporte de diferentes protocolos de HIIT no ha estudiado mayormente su combinación con otras estrategias, como las consejerías nutricionales en el estudio de la calidad de vida. Por ejemplo, estos parámetros en el HIIT se han estudiado en pacientes con cáncer ${ }^{15}$, y con enfermedad cardiovascular ${ }^{16}$, pero no existen reportes en pacientes con DM2. El objetivo del presente estudio fue determinar los efectos de 12 semanas de un programa HIIT, más consejería nutricional en variables cardiometabólicas y de calidad de vida en pacientes con DM2.

\section{Materiales y Métodos}

\section{Diseño}

En un estudio cuasiexperimental, con una muestra total de 21 pacientes mujeres con diabetes mellitus tipo $2(\mathrm{n}=21)$, de las cuales fueron incluidas pacientes insulino requirientes $(I R ; n=6)$ que no tenían diagnóstico de diabetes mellitus tipo 1 , y pacientes no insulino requirientes (NIR; $n=15)$, físicamente inactivas (menos de $150 \mathrm{~min}$ de $\mathrm{AF} /$ semana $)^{8}$, derivadas por medio del programa de salud cardiovascular (PSCV) por profesionales de cuatro centros de salud familiar (CESFAM) públicos (Padre las Casas, Las Colinas, Pulmahue, Conunhuenu) de la localidad de Padre las Casas, IX Región de la Araucanía.

Los sujetos $(n=21)$ fueron asignados a un grupo experimental (GE) con ejercicio HIIT + consejería nutricional, incluyéndose (GE: 3 IR y 8 sujetos NIR, $\mathrm{n}=11$ ), o a un grupo control (GC) solo con consejería nutricional, incluyéndose (GC: 3 IR y 7 sujetos NIR, $\mathrm{n}=10$ ). El tamaño de la muestra se calculó utilizando cambios observados en la glicemia (delta $=2,3 ; \mathrm{DE}=1,7$ $\mathrm{mg} / \mathrm{dl}$ ) en un grupo intervenido con similares intervenciones ${ }^{17}$. Un total de 9 participantes por grupo otorga una potencia de $80 \%$ y un error alfa 0,05 . Dos sujetos DM2-NIR $(\mathrm{n}=2)$ del GE fueron excluidos por no cumplir con la adherencia mínima $(80 \%)$. Finalmente, 19 sujetos $(\mathrm{n}=19)$ fueron incluidos en los análisis estadísticos. Las características de los pacientes son descritas en la Tabla 1.

Los criterios de inclusión incluyeron a) mujeres con diagnóstico de DM2; b) físicamente inactivas (menos de 150 min semanales) ${ }^{8}$; c) con hemoglobina glicosilada (HbAlc) > 6,5\% ${ }^{18}$; d) entre 40 y 65 años; e) índice de masa corporal $>25-35 \mathrm{~kg} / \mathrm{m}^{2}$; f) con control de profesional médico y electrocardiograma de reposo actualizado en el PSCV. Los criterios de exclusión fueron a) pacientes con problemas osteo-articulares; b) problemas derivados de diabetes (neuropatía periférica, retinopatía, nefropatía, heridas en los pies); c) arritmias (taquicardia, bradicardia); d) historial de infarto o accidente cerebrovascular, y h) recuperación post ejercicio menor a 15 latidos en 1 minuto. El estudio fue desarrollado considerando la Declaración de Helsinki, y fue aprobado por el Comité de Bioética del Servicio de Salud de la Red Araucanía Sur, Temuco-Chile.

\section{Evaluación de la composición corporal}

El peso $(\mathrm{kg})$ y talla $(\mathrm{m})$ se evaluaron con una balanza-tallímetro marca Detecto ${ }^{\circledR}$ (USA), con precisión de $0,1 \mathrm{~kg}$ y $0,1 \mathrm{~cm}$. El índice de masa corporal (IMC), se calculó mediante la fórmula $\left(\mathrm{kg}\right.$ de peso $\left./ \mathrm{m}^{2}\right)$. El porcentaje de grasa corporal se midió con un bioimpedanciómetro marca Beurer BF18 ${ }^{\circledR}$ (Alemania), similar a estudios previos $^{19}$. 


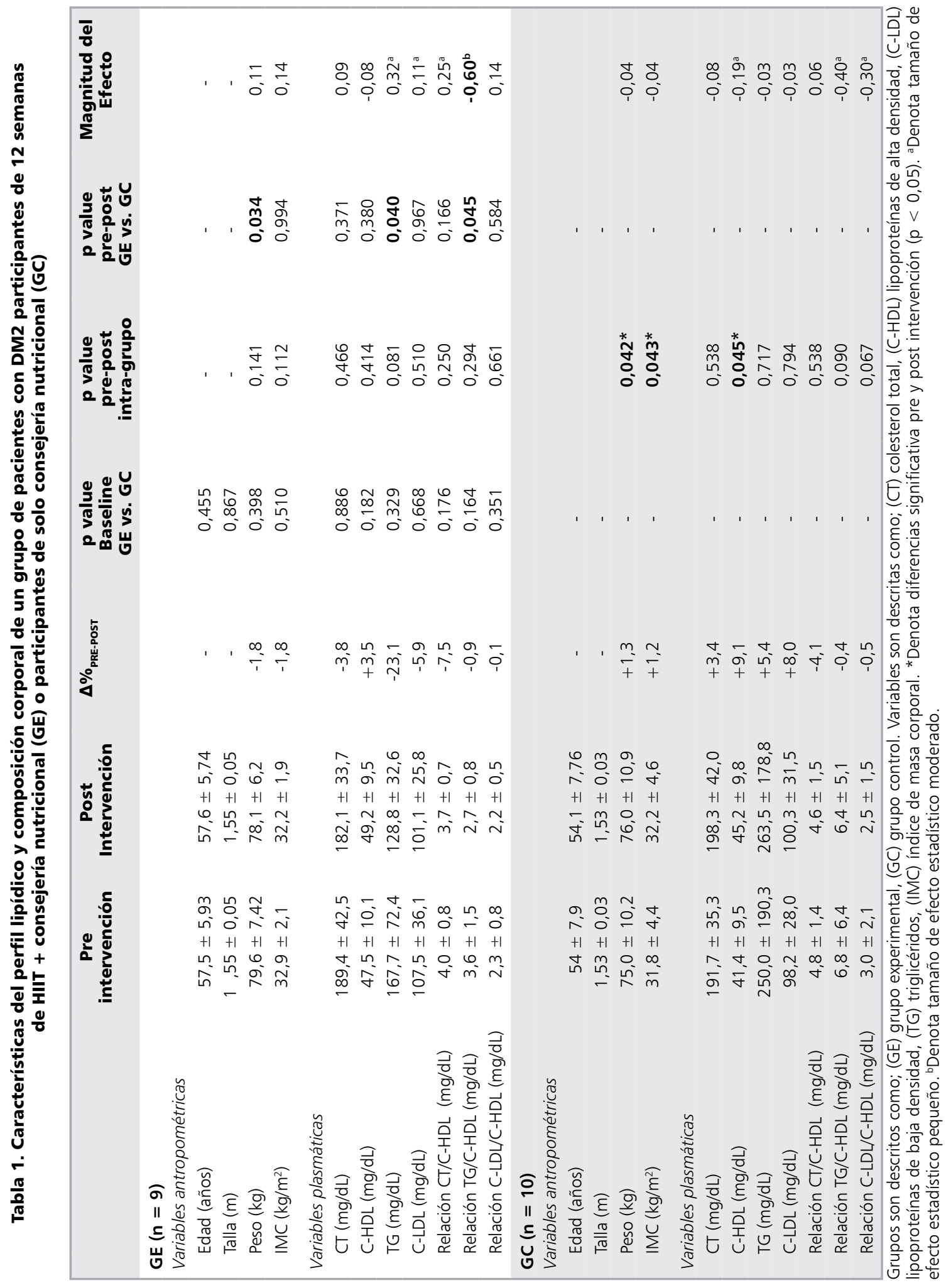




\section{Evaluación de la calidad de vida}

Se utilizó el cuestionario de calidad de vida SF-12, validado para la población chilena ${ }^{20}$ con una estimación de la fiabilidad mediante el alfa de Cronbach superior a 0,74 en ambas dimensiones (física y psicológica). El cuestionario consta de 12 ítems: función física (2 preguntas), rol físico (2 preguntas), dolor corporal (1 pregunta), salud general (1 pregunta), vitalidad (1 pregunta), rol emocional (2 preguntas), salud mental (2 preguntas), y función social (1 pregunta). El puntaje va entre 0 y $100 \%$, donde el mayor puntaje implica una mejor calidad de vida relacionada con la salud.

\section{Programa de ejercicio físico HIIT y consejería nutricional}

Antes de la intervención, los pacientes del GE realizaron una evaluación de tolerancia al ejercicio mediante la cuantificación de la resistencia máxima tolerada por cada individuo, a través del test de Astrand modificado y usando bicicletas estáticas marca Sports Arts ${ }^{\circledR}$, C521 U-R (Taiwán). Posteriormente, los sujetos participaron de una semana (3 sesiones en días alternados) de familiarización al HIIT. La intensidad de ejercicio (pedaleo) se controló a través de la escala de Borg adaptada (0-10), similar a otros estudios ${ }^{21}$, con la máxima resistencia $(100 \%)$, calculada previamente. Paralelamente, se midió la respuesta de la frecuencia cardiaca, reportándose esta de acuerdo al método de Karvonen ${ }^{22}$, utilizando un cardiómetro marca Polar $^{\circledR}$ T34 (Alemania). Las sesiones de HIIT se llevaron a cabo en el Centro Comunitario de Rehabilitación de Padre las Casas. Las características del programa HIIT son descritas en la Tabla 2.

Adicionalmente, siguiendo recomendaciones de instituciones públicas nacionales ${ }^{23}$, los sujetos del grupo GE participaron de una "consejería nutricional" en su respectivo centro de salud. La consejería consistió en reducir la ingesta de grasa saturada, azúcar e incrementar la ingesta de fibra. Esta consejería incluyó: eliminar azúcares simples, reducir porciones de carbohidratos complejos (aproximadamente $200 \mathrm{~g} /$ día) de bajo índice glicémico, fraccionados en 4 comidas diarias, con preferencia en alimentos altos en fibra (productos integrales, cereales integrales, frutas, verduras, legumbres). Se realizó 1 control médico y una consejería nutricional a los pacientes, de 20 a $30 \mathrm{~min}$, antes de la inclusión al protocolo de ejercicio. El grupo GC participó solo de la consejería nutricional de estilos de vida saludable.

\section{Evaluación de glicemia, hemoglobina glicosilada y perfil lipídico}

Las muestras de sangre venosa $(\sim 3,5 \mathrm{ml})$ fueron obtenidas después de $12 \mathrm{~h}$ de ayuno. Para el perfil lipídico (colesterol total [CT], lipoproteínas de baja densidad [C-LDL], lipoproteínas de alta densidad [C-HDL] y triglicéridos [TG]), HbAlc y glicemia las muestras se depositaron en un tubo con gel separador y activador de coagulación, se centrifugaron a $4.000 \mathrm{rpm}$ durante 10 min para obtención de suero. Para la HbAlc se

Tabla 2. Descripción del programa de ejercicio físico HIIT de 12 semanas

\begin{tabular}{|lcccc|}
\hline Variable & Semana 1-2-3 & Semana 4-5-6 & Semana 7-8-9 & Semana 10-11-12 \\
\hline Intensidad c/intervalo & Borg 7-8 & Borg 7-8 & Borg 7-8 & Borg 7-8 \\
\hline Duración c/intervalo (s) & 30 & 30 & 30 & 30 \\
\hline Recuperación (s) & 60 & 60 & 60 & 60 \\
\hline Método recuperación & Pasivo & Pasivo & Pasivo & Pasivo \\
\hline No intervalos & 20 & 20 & 20 & 20 \\
Cadencia (rpm) & $60-80$ & $60-80$ & $60-80$ & $60-80$ \\
Aumento 15 vatios (watt) & Semana 3 & Semana 6 & 30 & - \\
Volumen/día (min) & 30 & 30 & 90 & 30 \\
Volumen/semana (min) & 90 & 90 & $60-80$ & $60-80$ \\
FCR (\%) & $60-80$ & $60-80$ & & 90 \\
\hline
\end{tabular}

(rpm): Revoluciones por minuto, FCR: frecuencia cardiaca de reserva según Karvonen. 
utilizó una muestra sanguínea total depositadas en tubos con anticoagulante EDTA-K3 (Edta tripotásico), se utilizó 3,0 $\mathrm{ml}$ de muestra. Las muestras de suero para glicemia y perfil lipídico se analizaron inmediatamente obtenido el suero. Las muestras de $\mathrm{HbAlc}$ se mantuvieron a temperatura ambiente $\left(20{ }^{\circ} \mathrm{C}\right.$ aprox. $)$ hasta el análisis dentro del día. Para el análisis, tanto de glicemia, perfil lipídico y HbAlc se utilizó un equipo COBAS c501 del fabricante marca Roche/Hitachi ${ }^{\circledR}$ (Japón). La glicemia se analizó mediante el método enzimático de hexoquinasa. Para el CT se utilizó el método enzimático colorimétrico, utilizando CHOD-PAP. Para el C-HDL se utilizó sulfato de dextrano/enzimas modificadas con PEG (PEG-colesterol esterasa y PEG colesterol oxidasa). Para los TG, se utilizó el test enzimático colorimétrico utilizando GPOPOD. Para la HbA1c se utilizó el inmuno-ensayo turbidimétrico de inhibición (TINIA) para sangre total hemolizada, el resultado final se expresó en porcentaje según DCCT/NGSP.

\section{Análisis estadístico}

Se utilizó media \pm desviación estándar para describir las variables. Se calcularon los delta porcentual $(\Delta \%)$ entre pre y postintervención. Se utilizó el test de Shapiro-Wilk para analizar la normalidad y el test de Levene para establecer la homocedasticidad. Se utilizó el test $t$ de Student para establecer diferencias entre las medias de los grupos. Se utilizó ANOVA de medidas repetidas (grupos $\mathrm{x}$ tiempo), así como el post hoc de Bonferroni para localizar las diferencias entre los tiempos de medición. Se aplicó el coeficiente de correlación $r$ de Pearson para conocer la asociación entre los cambios en grasa y la HbAlc. Adicionalmente, para conocer la magnitud del efecto estadístico, se utilizó la prueba d de Cohen, considerando $0,20,0,60,1,2$, y 2,0 como efecto pequeño, moderado, alto y muy alto, respectivamente. El nivel para significancia estadística se estableció en $\mathrm{p}<0,05$. Todos los análisis estadísticos se realizaron utilizando el software SPSS (versión 18.0, Chicago, Illinois, USA).

\section{Resultados}

Al inicio de la intervención no existieron diferencias significativas en la línea de base entre las variables (Tabla 1, Tabla 2, Figura 1, Figura 2).

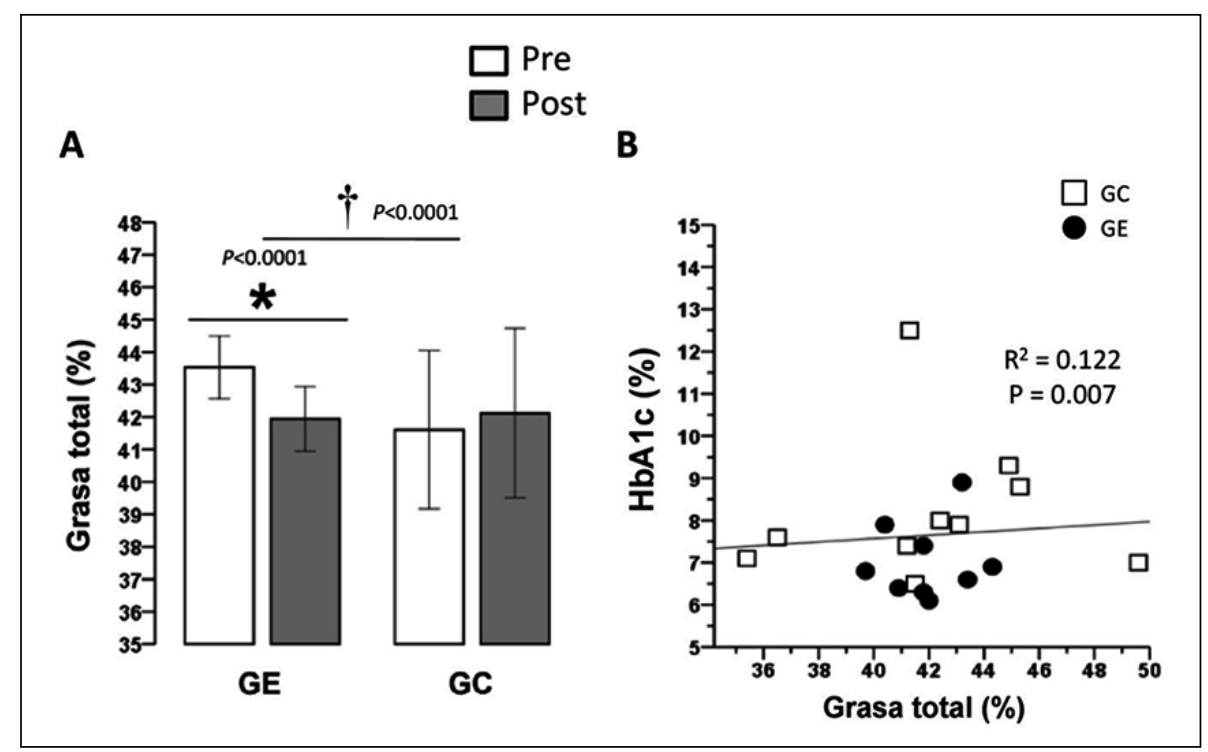

Figura 1. Cambios en el porcentaje de grasa total después de 12 semanas de intervención con ejercicio HIIT + consejería nutricional $(\mathbf{A})$ y $(\mathbf{B})$ correlación entre los cambios en la grasa total y los cambios en la HbA1c después de intervención. Grupos son descritos como (GE) grupo experimental, (GC) grupo control. *Denota cambios o/ correlación estadísticamente significativos (a) a nivel $p<0,05$. †Denota diferencias significativas entre grupos a nivel $(p<0,0001)$. 


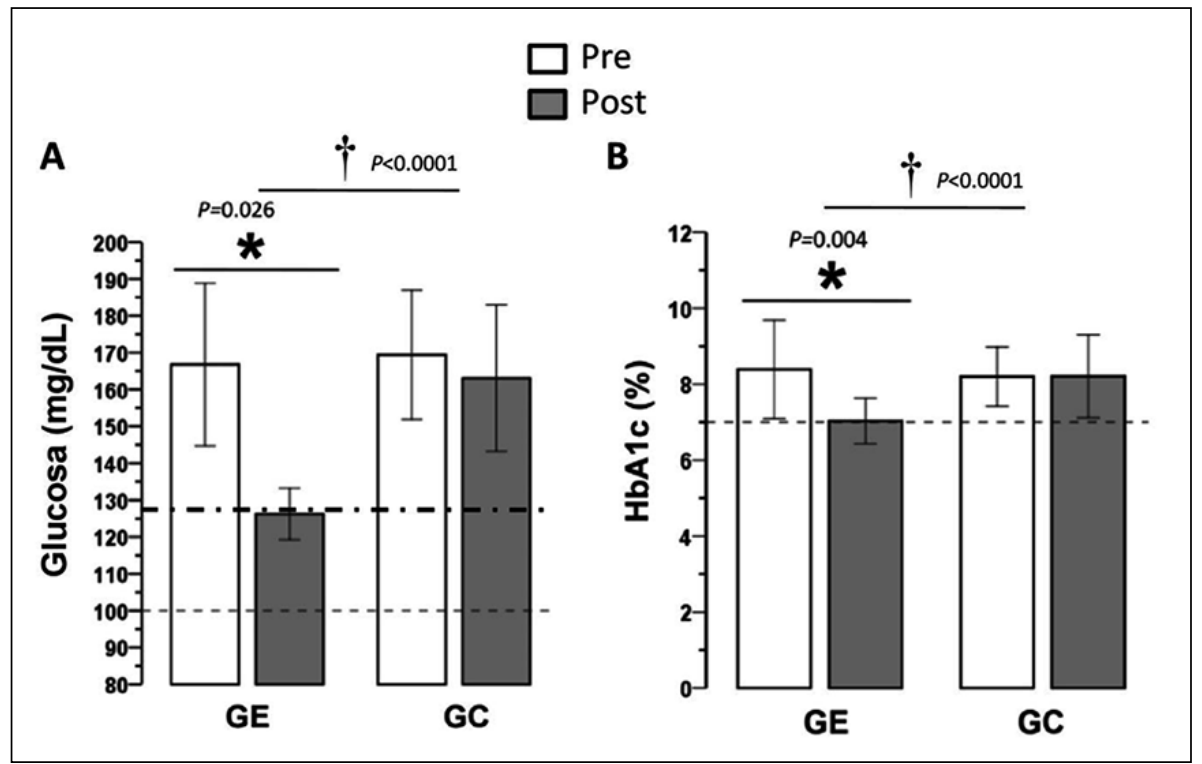

Figura 2. Cambios en la glucosa en ayunas (A), y (B) cambios en la hemoglobina glicosilada (HbA1c) después de 12 semanas de intervención con ejercicio tipo HIIT + consejería nutricional. Grupos son descritos como (GE) grupo experimental, (GC) grupo control. (---) Denota valores límites para normoglicémico/hiperglicemico en $(\mathbf{A})$ y en $(\mathbf{B})$ denota valores recomendables de $\mathrm{HbA} 1 \mathrm{c}$ para pacientes con diabetes. (---) Denota valores límites para hipergicémico/DM2. *Denota cambios estadísticos significativos test a nivel $p<0,05$. †Denota diferencias significativas entre grupos a nivel $(p<0,0001)$.

En el grupo GE existieron cambios significativos $(\mathrm{p}<0,05)$ después de intervención en los niveles de porcentaje de grasa, desde $43,5 \pm 1,5$ a $41,9 \pm 1,5 \%, \mathrm{p}<0,0001$ (Figura 1A), en la glicemia desde 166,8 $\pm 66,2$ a $126,2 \pm 21,1 \mathrm{mg} / \mathrm{dl}, \mathrm{p}=0,026$ (Figura 2A), así como en la HbAlc desde 8,4 $\pm 1,9$ a $7,0 \pm 0,9 \%, p=0,004$, (Figura $2 B$ ). No existieron cambios en la grasa (Figura 1A), glicemia (Figura 2A) y HbA1c (Figura 2B) en el grupo GC. Entre grupos (GE vs GC), existieron diferencias significativas en los cambios en grasa, glicemia y HbAlc, (Figura 1A, Figura 2A y Figura 2B). Se presentó una correlación significativa entre la reducción del porcentaje de grasa total vs la reducción de la HbAlc $\mathrm{R}^{2}=0,122$ (Figura 1B).

En el grupo GE no existieron cambios significativos pre-postintervención en el CT, C-LDL, C-HDL, TG, y en las relaciones CT/C-HDL, TG/CHDL, C-LDL/C-HDL (Tabla 1). Se observó un incremento significativo en el C-HDL, peso e IMC en el grupo GC (Tabla 1). Se observó un cambio estadístico pequeño en la magnitud del efecto postintervención en la variable TG y C-LDL del grupo GE, donde el promedio en la variable TG se redujo a parámetros normales clínicos $(<150$ $\mathrm{mg} / \mathrm{dl}$ ) postintervención (Tabla 1). Entre grupos (GE vs GC), se registró una diferencia significativa en los cambios después de intervención en los triglicéridos, y en la relación TG/C-HDL (Tabla 1). En el grupo GE existieron cambios significativos (benéficos) en todos los ítems en delta porcentual $\Delta \%_{\text {PRE-POST }}$ (función física $+141,7 \%$, rol físico $+68,5 \%$, dolor $+70,0 \%$, salud general $+125,6 \%$, vitalidad $+128,8 \%$, rol emocional $+51,0 \%$, salud mental $+13,5 \%$ y función social $+47,9 \%$ ) relacionados con la calidad de vida (Tabla 3 ).

\section{Discusión}

Los principales hallazgos del estudio indican que en comparación a una condición control (consejería nutricional aislada), 12 semanas de ejercicio HIIT + consejería nutricional mejoran no solo parámetros de la salud cardiometabólica (reducción de la grasa, glicemia, HbAlc), sino también parámetros relacionados con la calidad de vida de pacientes con diabetes. 
Tabla 3. Cambios en parámetros de calidad de vida después de intervención con ejercicio tipo HIIT + consejería nutricional (GE) o sólo consejería nutricional (GC)

\begin{tabular}{|c|c|c|c|c|c|}
\hline & $\begin{array}{c}\text { Pre } \\
\text { intervención }\end{array}$ & $\begin{array}{c}\text { Post } \\
\text { intervención }\end{array}$ & $\Delta \%_{\text {PRE-POST }}$ & $\begin{array}{l}\text { p value } \\
\text { pre-post }\end{array}$ & $\begin{array}{l}\text { Magnitud } \\
\text { del efecto }\end{array}$ \\
\hline \multicolumn{6}{|l|}{$\mathrm{GE}(n=9)$} \\
\hline \multicolumn{6}{|c|}{ Cuestionario de Calidad de Vida } \\
\hline Función física & $33,3 \pm 27,9$ & $80,5 \pm 20,8$ & $+141,7$ & $<0,001 *$ & $-0,69^{b}$ \\
\hline Rol físico & $52,7 \pm 25,6$ & $88,8 \pm 11,6$ & $+68,5$ & $<0,001 *$ & $-0,67^{b}$ \\
\hline Dolor & $55,5 \pm 32,5$ & $94,4 \pm 20,8$ & $+70,0$ & $<0,01^{*}$ & $-0,58^{a}$ \\
\hline Salud general & $22,2 \pm 19,5$ & $50,1 \pm 12,5$ & $+125,6$ & $<0,001^{*}$ & $-0,64^{b}$ \\
\hline Vitalidad & $38,8 \pm 33,3$ & $88,8 \pm 13,1$ & $+128,8$ & $<0,001^{*}$ & $-0,71^{b}$ \\
\hline Rol emocional & $59,7 \pm 25,6$ & $90,2 \pm 8,3$ & $+51,0$ & $<0,001 *$ & $-0,62^{b}$ \\
\hline Salud mental & $47,2 \pm 12,1$ & $53,61 \pm 14,5$ & $+13,5$ & $<0,01^{*}$ & $-0,66^{b}$ \\
\hline Función social & $63,8 \pm 28,2$ & $94,4 \pm 11,1$ & $+47,9$ & $0,02 *$ & $-0,58^{a}$ \\
\hline \multicolumn{6}{|l|}{$\mathrm{GC}(n=10)$} \\
\hline \multicolumn{6}{|c|}{ Cuestionario de Calidad de Vida } \\
\hline Función física & $57,5 \pm 26,5$ & $55,0 \pm 30,7$ & $-4,3$ & 0,594 & 0,04 \\
\hline Rol físico & $67,5 \pm 23,7$ & $65,0 \pm 21,1$ & $-3,7$ & 0,595 & 0,05 \\
\hline Dolor & $65,0 \pm 37,6$ & $52,5 \pm 32,2$ & $-19,2$ & 0,243 & 0,17 \\
\hline Salud general & $32,5 \pm 16,9$ & $27,5 \pm 18,4$ & $-15,3$ & 0,175 & 0,14 \\
\hline Vitalidad & $55,0 \pm 19,7$ & $57,5 \pm 26,5$ & $+4,5$ & 0,597 & $-0,05$ \\
\hline Rol emocional & $68,8 \pm 23,0$ & $71,3 \pm 26,4$ & $+3,6$ & 0,591 & $-0,05$ \\
\hline Salud mental & $45,0 \pm 25,8$ & $40,0 \pm 22,7$ & $-11,1$ & 0,171 & 0,11 \\
\hline Función social & $70,0 \pm 28,4$ & $72,5 \pm 32,2$ & $+3,5$ & 0,596 & $-0,04$ \\
\hline
\end{tabular}

Grupos son descritos como; (GE) grupo experimental, (GC) grupo control. Cambios estadísticos son descritos como; *Denota diferencias significativas entre pre y post intervención $(p<0,05)$. ${ }^{a, b}$ denota tamaño del efecto estadístico pequeños y moderados respectivamente.

Interesantemente, en todas las áreas evaluadas de la calidad de vida monitoreadas a través del cuestionario SF-12 se pueden encontrar diferencias significativas entre antes y después de intervención en el grupo GE (Tabla 3). Por ejemplo, en este grupo, la función física se incrementó en $+141,7 \%$, así como la salud general $+125,6 \%$ y la percepción de vitalidad $+128,8 \%$ de estos pacientes (Tabla 3). Al respecto, nosotros creemos que estos hallazgos podrían tener relación con el incremento de la fuerza y potencia en los músculos inferiores que se ejercitan durante HIIT en bicicleta, donde se ha reportado que sujetos bajo este régimen de ejercicio incrementan la condición muscular ${ }^{24}$. En este sentido, y atendiendo a la capacidad de adaptación muscular con ejercicio, nosotros incrementamos cada 3 semanas la carga de "pedaleo" en bicicleta en 15 vatios, donde, en promedio, los sujetos del grupo GE terminaron ejercitándose con más de 45 watts de potencia adicionados, respecto de la línea de base con la que iniciaron.

Es importante recordar que la característica más importante del ejercicio HIIT es incrementar rápidamente la insulino sensibilidad ${ }^{25}$ y reducir los niveles de grasa corporal ${ }^{12}$, mediante sus mecanismos de activación adrenérgica durante y posterior al ejercicio ${ }^{13}$. Adicionalmente, nuestro estudio reporta nueva información en parámetros de calidad de vida, donde, si bien es cierto existieron cambios significativos en todos los ítems del cuestionario SF-12, el rol emocional, la salud mental y la función social registraron menores cambios (Tabla 3), por lo tanto, se podría inferir que estos 3 componentes pueden depender de numerosas variables donde no precisamente el ejercicio y la consejería nutricional juegan un rol directo. En otros estudios, 8 semanas de HIIT en pacientes con enfermedad isquémica demostraron mejorar su calidad de vida mediante ejercicio continuo, 
aunque solo HIIT produjo mejores efectos benéficos a nivel de morbilidad ${ }^{16}$.

Respecto a los cambios en el control glicémico, es importante mencionar que estudios aplicando aisladamente solo HIIT han reportado similares efectos benéficos en el control glicémico ${ }^{10,12,26} \mathrm{y}$ composición corporal ${ }^{13}$. Después de 16 semanas de HIIT, Álvarez y cols, redujeron la glicemia en -14\% en pacientes con DM2 $2^{10}$. En 12 semanas de HIIT, los mismos autores ${ }^{27}$ redujeron $-19 \%$ la glicemia de pacientes hiperglicémicos. Por otra parte, en solo 2 semanas de HIIT, Whyte y cols. ${ }^{26}$ demostraron reducción la glicemia en $-5 \%$ en adultos con sobrepeso/obesidad. Interesantemente, en los estudios de HIIT, aunque no siempre se han apreciado reducciones importantes en el peso corporal total, frecuentemente HIIT reduce la grasa corporal. Por ejemplo, Mancilla y cols, ${ }^{12}$ redujeron $-4,3 \mathrm{~kg}$ (12 semanas HIIT) de grasa corporal y el perímetro de cintura en $-2,3 \mathrm{~cm}$. Otros estudios han reportado, en el mismo tiempo, incluso una reducción de $-6 \mathrm{~cm}$ en este marcador bajo solo régimen de ejercicio HIIT $^{27}$.

Por otra parte, posterior a la finalización del estudio fue necesaria una reevaluación de los pacientes en su farmacoterapia, siendo necesaria una 'regulación' de las dosis de fármacos en algunos de los pacientes del grupo GE, en las unidades de metformina, glibenclamida, insulina protamina neutra, de Hagedorn, atorvastatina, e hidroclorotiazida (datos no mostrados), de acorde a como previamente se ha reportado ${ }^{14}$. En estudios previos, Cade y cols., después de 12 semanas de ejercicio caminando 2 millas/día, suspendieron la farmacoterapia de 24 pacientes con hipertensión arterial quienes disminuyeron su presión sistólica en -22 y diastólica en $-18 \mathrm{mmHg}^{28}$. Recientemente, Cano y cols. ${ }^{14}$ reportaron importantes reducciones en las unidades/día de diferentes fármacos relacionados al control glicémico y de la presión arterial.

Entre algunas fortalezas y debilidades, se puede mencionar que la inclusión en la evaluación de la calidad de vida agrega información no reportada a los conocidos efectos cardiometabólicos del HIIT en pacientes con DM2. Sin embargo, una debilidad del estudio, fue que la muestra no fue randomizada.

En conclusión, 12 semanas de ejercicio tiempo-eficiente como HIIT + consejería nutricional mejoran no solo parámetros de la salud cardiometabólica, sino también marcadores de calidad de vida, en comparación a solo la aplicación de consejería nutricional de pacientes mujeres con DM2 pertenecientes a los PSCV del sistema de salud público chileno.

Agradecimientos: Queremos agradecer a las (os) profesionales del PSCV de los CESFAM (E. Rosales, G. Muñoz, M. Gómez), al Sr. Danner Burgos, Director CCR, nutricionistas, Laboratorio Clínico (S. Huaiquimil, A. Lagos), Departamento de Salud, y a la Ilustre Municipalidad de Padre las Casas.

\section{Referencias}

1. Danaei G, Finucane MM, Lu Y, Singh GM, Cowan MJ, Paciorek CJ, et al. National, regional, and global trends in fasting plasma glucose and diabetes prevalence since 1980: systematic analysis of health examination surveys and epidemiological studies with 370 country-years and 2. 7 million participants. Lancet 2011; 378 (9785): 31-40.

2. Cheng YJ, Imperatore G, Geiss LS, Wang J, Saydah SH, Cowie CC, et al. Secular changes in the age-specific prevalence of diabetes among US adults: 1988-2010. Diabetes Care 2013; 36 (9): 2690-6.

3. Celis-Morales C, Salas C, Alduhishy A, Sanzana R, Martínez MA, Leiva A, et al. Socio-demographic patterns of physical activity and sedentary behaviour in Chile: results from the National Health Survey 2009-2010. J Public Health (Oxford, Engl) 2015: 1-8.

4. MINSAL. Ministerio de Salud. Gobierno de Chile. Encuesta Nacional de Salud ENS Chile 2009-2010. Disponible en: http://www.redsalud.gov.cl/portal/url/ item/99c12b89738d80d5e04001011e0113f8.pdf 2011.

5. Celis-Morales C, Salas C, Álvarez C, Aguilar Farías N, Ramírez-Campillos R, Leppe J, et al. Un mayor nivel de actividad física se asocia a una menor prevalencia de factores de riesgo cardiovascular en Chile: resultados de la Encuesta Nacional de Salud 2009-2010. Rev Med Chile 2015; 143: 1435-43.

6. Fang ZY, Sharman J, Prins JB, Marwick TH. Determinants of Exercise Capacity in Patients With Type 2 Diabetes. Diabetes Care 2005; 28 (7): 1643-8.

7. Colberg SR, Sigal RJ, Fernhall B, Regensteiner JG, Blissmer BJ, Rubin RR, et al. Exercise and Type 2 Diabetes: The American College of Sports Medicine and the American Diabetes Association: joint position statement. Diabetes Care 2010; 33 (12): e147-67.

8. Ministerio del Deporte, Gobierno de Chile, 2016. Política Nacional de Actividad Física y Deporte 2016-2025. 
Subsecretaria de deporte, División de política y gestión deportiva. Área de Políticas Públicas. $1^{\text {era }}$ Edición, Santiago, Chile.

9. Burgomaster KA, Howarth KR, Phillips SM, Rakobowchuk M, MacDonald MJ, McGee SL, et al. Similar metabolic adaptations during exercise after low volume sprint interval and traditional endurance training in humans. J Physiol 2008; 586 (1): 151-60.

10. Álvarez C, Ramírez-Campillo R, Martínez-Salazar C, Mancilla R, Flores-Opazo M, Cano-Montoya J, et al. Low-Volume High-Intensity Interval Training as a Therapy for Type 2 Diabetes. Int J Sports Med 2016; 37 (09): 723-9.

11. Little JP, Gillen JB, Percival ME, Safdar A, Tarnopolsky MA, Punthakee Z, et al. Low-volume high-intensity interval training reduces hyperglycemia and increases muscle mitochondrial capacity in patients with type 2 diabetes. J Appl Physiol 2011; 111 (6): 1554-60.

12. Mancilla R, Torres P, Álvarez C, Schifferli I, Sapunar J, Díaz E. [High intensity interval training improves glycemic control and aerobic capacity in glucose intolerant patients]. Rev Med Chile 2014; 142 (1): 34-9.

13. Boutcher SH. High-intensity intermittent exercise and fat loss. J Obes 2011; 2011: 868305.

14. Cano-Montoya J, Ramírez-Campillo R, Martínez C, Sade-Calles F, Salas-Parada A, Álvarez C. Interacción entre farmacoterapia hipotensiva y terapia con ejercicio físico requiere regulación farmacológica en pacientes hipertensos. Rev Med Chile 2016; 144 (2): 152-61.

15. Toohey K, Pumpa KL, Arnolda L, Cooke J, Yip D, Craft PS, et al. A pilot study examining the effects of low-volume high-intensity interval training and continuous low to moderate intensity training on quality of life, functional capacity and cardiovascular risk factors in cancer survivors. PeerJ 2016; 4: e2613.

16. Jaureguizar KV, Vicente-Campos D, Bautista LR, de la Peña CH, Gómez MJA, Rueda MJC, et al. Effect of High-Intensity Interval Versus Continuous Exercise Training on Functional Capacity and Quality of Life in Patients With Coronary Artery Disease: A Randomized Clinical Trial. J Cardiopulm Rehab Prev 2016; 36 (2): 96-105.

17. Álvarez C, Ramírez R, Flores M, Zúñiga C, Celis-Morales CA. Efectos del ejercicio físico de alta intensidad y sobrecarga en parámetros de salud metabólica en mujeres sedentarias, pre-diabéticas con sobrepeso u obesidad.
Rev Med Chile 2012; 140: 1289-96.

18. Colberg SR, Albright AL, Blissmer BJ, Braun B, Chasan-Taber L, Fernhall B, et al. Exercise and type 2 diabetes: American College of Sports Medicine and the American Diabetes Association: joint position statement. Exercise and type 2 diabetes. MSSE 2010; 42 (12): 2282-303.

19. Jebb SA, Cole TJ, Doman D, Murgatroyd PR, Prentice AM. Evaluation of the novel Tanita body-fat analyser to measure body composition by comparison with a four-compartment model. Br J Nutr 2000; 83 (02): 11522.

20. Vera-Villarroel P, Silva J, Celis-Atenas K, Pavez P. Evaluación del cuestionario SF-12: verificación de la utilidad de la escala salud mental. Rev Med Chile 2014; 142 (10): 1275-83.

21. Ciolac EG, Mantuani SS, Neiva CM. Rating of perceived exertion as a tool for prescribing and self regulating interval training: a pilot study. Biol Sport 2015; 32 (2): 103-8.

22. Karvonen J, Vuorimaa T. Heart rate and exercise intensity during sports activities. Practical application. Sports Med 1988; 5 (5): 303-11.

23. MINSAL. Manejo alimentario del adulto con sobrepeso u obesidad. In: cardiovascular Pds, editor. Santiago 2002.

24. Martin DT, Scifres JC, Zimmerman SD, Wilkinson JG. Effects of Interval Training and a Taper on Cycling Performance and Isokinetic Leg Strength. Int J Sports Med 1994; 15 (8): 485-91.

25. Hawley JA, Lessard SJ. Exercise training-induced improvements in insulin action. Acta Physiol 2008; 192 (1): 127-35.

26. Whyte LJ, Gill JMR, Cathcart AJ. Effect of 2 weeks of sprint interval training on health-related outcomes in sedentary overweight/obese men. Metab 2010; 59 (10): 1421-8.

27. Álvarez C, Ramírez-Campillo R, Flores-Opazo M, Henríquez-Olguín C, Campos C, Carrasco V, et al. [Metabolic response to high intensity exercise training in sedentary hyperglycemic and hypercholesterolemic women]. Rev Med Chile 2013; 141: 1293-9.

28. Cade R, Mars D, Wagemaker H, Zauner C, Packer D, Privette $\mathrm{M}$, et al. Effect of aerobic exercise training on patients with systemic arterial hypertension. Am J Med 1984; 77 (5): 785-90. 